\title{
IjEHSR
}

\section{Metadata Analysis}

\section{Metadata analysis of the squalene epoxidase gene in dermatophytes.}

\section{Fatima Ismail (D), Iqra Ahmad (D) \& Eqaza Javed}

Department of Biochemistry and Biotechnology, The Islamia University of Bahawalpur, Bahawalpur-Pakistan.

Doi: 10.29052/JJEHSR.v9.11.2021.129-142

Corresponding Author Email:

fatima.ismail@ymail.com

Received 01/10/2020

Accepted 25/01/2021

First Published 01/03/2021

\section{(c) (C)}

(C) The Author(s). 2021 Open Access This article is distributed under the terms of the Creative Commons Attribution 4.0 International License (http://creativecommons.org/licenses/by/4.0/)

\section{Abstract}

Background: Squalene epoxidase gene is an azole antifungal target enzyme in the ergosterol biosynthesis pathway in fungi-the inhibition of enzyme aggregate squalene, a type of ergosterol derivative depletion that leads to fungal growth inhibition. Squalene epoxidase widely diverges in three fungal groups of dermatophytes Trichophyton, Microsporum and Epidermophyton.

Methodology: The study has demonstrated a variety of squalene epoxidase genes in the dermatophyte genome. Squalene epoxidase gene was also studied for possible single nucleotide polymorphism (SNPs) in the Trichophyton group's homologs and found multiple SNP positions that induce non-synonymous mutation and change the sequence of amino acid into protein structure that can change phenylalanine to leucine.

Results: Squalene epoxidase is widely present in dermatophytes. Mutation in the squalene epoxidase gene can mislead normal sterol formation in the fungal cell membrane that induces fungal resistance against several drugs, including azoles and polyenes. Squalene epoxidase gene carries 2 - 3 transcripts and 2 - 3 number of exons. Squalene epoxidase gene has FAD-dependent oxidoreductases and NADP binding domain conserved domains in fungal groups of dermatophytes.

Conclusion: This study analyzed the abundance of the squalene epoxidase gene, suggesting that squalene epoxidase gene resistant mutants can occur naturally. Thus squalene epoxidase gene should be extensively studied in order to increase the potential of available antifungals.

\section{Keywords}

Squalene Epoxidase, Resistant Mutant, Trichophyton, Microsporum, Epidermophyton.

\section{Check for updates}




\section{Introduction}

Dermatophytes are a very specialized group of keratinophilic fungi responsible for superficial mycoses of humans and animals ${ }^{1}$. Dermatophyte infections are a worldwide major health concern, specifically in an immunocompromised individual. They may pose severe risks. Dermatophytes are divided into three genera: Microsporum, Trichophyton and Epidermophyton, although the precise taxonomic associations of this fungal group are far from being fully determined. Regardless of the common occurrence of dermatophyte infections, little is known about these pathogens' molecular biology. Dermatophytes are slowgrowing as compare to other more significant fungal pathogens. They are not very well studied for their metabolic reactions and stress adaptive mechanisms. However, several natural mutants are investigated in clinical and agricultural practices ${ }^{2}$.

Squalene epoxidase is a monooxygenase that catalyzes the conversion of squalene to 2, 3oxidosqualene. It is a microsomal membraneassociated enzyme that functions as a significant regulator in the biosynthetic pathway of sterols. Ergosterol helps in the photo-oxidation of fungi. This enzyme needs oxygen, Nicotine amide, Adenine dinucleotide, Phosphate hydrogen and Flavin adenine dinucleotide for stimulation ${ }^{3}$. Previously, terbinafine resistant isolates have been reported in increasing number. Terbinafine inhibits the Squalene epoxidase (SE) enzyme and interferes with the ergosterol biosynthesis, an integral cell membrane constituent. Mutations have been identified within the squalene epoxidase gene like clinical terbinafine resistant isolates ${ }^{4}$.

Squalene epoxidase gene is involved in ergosterol biosynthesis, which converts squalene to lanosterol in sterol biosynthesis. This enzyme functions as a major regulator in the biosynthetic pathway to sterols $s^{5}$. Mutation in this gene causes resistance against fluconazole, ketoconazole, itraconazole, voriconazole and terbinafine drugs because of the modification of the target enzyme by mutation induces increased drug efflux, stress adaptation and overexpression of drug efflux, which degrade the drug effects ${ }^{6}$.
Squalene epoxidase is an enzyme involved in the early steps of ergosterol biosynthesis, is inhibited by allylamines such as terbinafine. This inhibition contributes to squalene accumulation that is toxic for fungi. Resistance is due to single point mutations in the gene encoding squalene epoxidase. Both mutations introduced missense replacements of amino acids (Leu393Phe in one case and Phe397Leu in the other), leading to 100 fold higher $\mathrm{MIC}^{7}$. Terbinafine resistant mutants of Trichophyton rubrum, Aspergillus nidulans and Aspergillus fumigatus strains were identified. This mutant carries a mutation in the squalene epoxidase gene that contributes to the replacement of leucine by phenylalanine in the mutant of $\mathrm{Erg}^{8}{ }^{8}$.

ERG1 (squalene epoxidase) protein comprised of two flavin adenine dinucleotide domains (FAD) and one nucleotide-binding domain (NB). ERG1 required oxygen from energy molecules, NADPH and FAD. ERG1 protein alignment with other squalene epoxidase showed two strongly conserved FAD-binding domains, FAD1 and FAD $2^{10}$. Spontaneous $P C R$ mutagenesis of the ERG1 gene identified one ERG1 allele that brings a mutation. It contributes to a single exchange of amino acids in the FAD1 domain near the ERG1 Nterminus ${ }^{11}$.

\section{Methodology}

\section{Squalene epoxidase gene sequence}

Squalene epoxidase gene from the dermatophytic fungi Trichophyton rubrum, Trichophyton mentagrophytes, Trichophyton interdigitale, Microsporum canis, Aspergillus Versicolor, Aspergillus flavus, Aspergillus fumigatus, Aspergillus niger, Candida albicans and Candida glabrata obtained from NCBI gene sequences. Squalene epoxidase and related proteins were explored within acquired amino acid sequences of a FAD-binding domain. In the next step, FADbinding proteins were searched against a transmembrane domain NADB Squalene epoxidase contains a putative FAD-binding site and is a crucial enzyme in the sterol biosynthetic pathway. Putative transmembrane regions are found in the proteins $\mathrm{C}$-terminus. For the detection 
of some unpredicted genes, we explored raw genomic sequences using NCBI nucleotide blast. Sequences of all known Squalene epoxidase genes were disguised from genomic sequences. All amino acid sequences of previously identified Squalene epoxidase genes were used as queries in nucleotide sequence blast.

For all Trichophyton species, similarity ranges from 44.66\%-45.34\%. Query cover ranges from 95\%99\%. For all Microsporum and Aspergillus species, similarity ranges from $37.34 \%-45.53 \%$. The range of query cover is $84 \%-99 \%$.

\section{Squalene epoxidase protein prediction}

The number of genes contained FAD and NABD binding domain, which was similar in all species of dermatophytes. These domains have an alphabeta-alpha configuration. NAD binding involves numerous hydrogen and van der Waals contacts.

\section{Transcript Exon and intron analysis}

Several exons and introns of different species of dermatophytes were obtained from NCBI. A single gene can produce multiple different RNAs (transcripts). Similarly, the numbers of transcripts were also obtained from the $\mathrm{CBI}$ database (https://www.ncbi.nlm.nih.gov/).

\section{Squalene epoxidase gene Phylogenetic Analysis}

A sequence alignment was done with ClustalX. Squalene epoxidase genes were determined by a conserved domain database. Phylogenetic analysis was made in MEGA6. Phylogenetic analysis was done using maximum possibility and neighbourhood joining method.

\section{Single nucleotide polymorphism}

Single nucleotide polymorphism (SNPs) analysis was found in the Squalene epoxidase gene of the Trichophyton group of dermatophytes. SNPs were obtained from $\mathrm{NCBI}$ and compared with the reported.

\section{Results \\ Bioinformatics survey of Squalene epoxidase gene}

The squalene epoxidase gene was analyzed using different bioinformatics tools. The comparison was made based on percentage similarity, exon count, conserved domains and query cover. Sequences were aligned, and phylogenetic trees were constructed. The squalene epoxidase gene in dermatophytes has an average sequence length of 489 amino acids.

\section{Squalene epoxidase protein prediction}

NAD binding involves numerous hydrogen-bonds and van der Waals contacts, particularly $\mathrm{H}$-bonding of residues in turn between the first strand and the subsequent helix of the Rossmann-fold topology. Characteristically, this turn exhibits a consensus binding pattern. The first 2 glycines participate in $N A D(P)$-binding, and the third facilitate close packing of the helix to the beta-strand (https://go.usa.gov/XARNY).

\section{Prediction of conserved domains of Squalene epoxidase}

In this study, we used genomic sequences of all available dermatophyte species. The first step is the identification of all Squalene epoxidase genes within their genomes. Squalene epoxidase consists of two types of domain. FAD and NADB. The identified genes were containing FAD and NABD binding domain. The $E$ value of 1 e-135 was used. Query cover ranges from 95\% to 99\%. The length of the amino acid sequence was $489 \mathrm{bp}$. Percentage similarity was very low such as $45.34 \%$ for Trichophyton rubrum. Trichophyton rubrum has the highest percentage similarity to other Trichophyton species, as shown in table 1. 
Table 1: Paralogs of Squalene epoxidase gene

\begin{tabular}{|c|c|c|c|c|c|c|c|}
\hline $\begin{array}{l}\text { S. } \\
\text { no }\end{array}$ & Species & Accession no & $\begin{array}{l}\text { Query } \\
\text { cover }\end{array}$ & $\begin{array}{c}\text { E- } \\
\text { value }\end{array}$ & $\begin{array}{l}\text { Percentage } \\
\text { similarity }\end{array}$ & $\begin{array}{l}\text { Amino } \\
\text { acid } \\
\text { sequence } \\
\text { length }\end{array}$ & Conserved domains \\
\hline 1 & $\begin{array}{l}\text { squalene } \\
\text { epoxidase } \\
\text { [Trichophyton } \\
\text { mentagrophytes] }\end{array}$ & APQ47162.1 & $99 \%$ & $5 e-136$ & 44.90 & 489 & $\begin{array}{l}\text { FAD-dependent } \\
\text { oxidoreductase, NADP } \\
\text { binding proteins }\end{array}$ \\
\hline 2 & $\begin{array}{l}\text { squalene } \\
\text { epoxidase } \\
\text { [Trichophyton } \\
\text { mentagrophytes] }\end{array}$ & ATA67033.2 & $99 \%$ & $7 e-136$ & 44.90 & 489 & $\begin{array}{l}\text { FAD-dependent } \\
\text { oxidoreductase, NADP } \\
\text { binding proteins }\end{array}$ \\
\hline 3 & $\begin{array}{l}\text { squalene } \\
\text { epoxidase } \\
\text { [Trichophyton } \\
\text { mentagrophytes] }\end{array}$ & QIJ32792.1 & $99 \%$ & $1 e-135$ & 44.90 & 489 & $\begin{array}{l}\text { FAD-dependent } \\
\text { oxidoreductase, NADP } \\
\text { binding proteins }\end{array}$ \\
\hline 4 & $\begin{array}{l}\text { squalene } \\
\text { epoxidase } \\
\text { [Trichophyton } \\
\text { mentagrophytes] }\end{array}$ & QIJ32790.1 & $99 \%$ & $4 e-135$ & 44.90 & 489 & $\begin{array}{l}\text { FAD-dependent } \\
\text { oxidoreductase, } \\
\text { NADP binding } \\
\text { proteins }\end{array}$ \\
\hline 5 & $\begin{array}{l}\text { squalene } \\
\text { epoxidase } \\
\text { [Trichophyton } \\
\text { mentagrophytes] }\end{array}$ & QIJ32789.1 & $99 \%$ & $5 e-135$ & 44.69 & 489 & $\begin{array}{l}\text { FAD-dependent } \\
\text { oxidoreductase, NADP } \\
\text { binding proteins }\end{array}$ \\
\hline 6 & $\begin{array}{l}\text { squalene } \\
\text { epoxidase } \\
\text { [Trichophyton } \\
\text { mentagrophytes] }\end{array}$ & ATA67030.2 & $99 \%$ & $6 e-135$ & 44.69 & 489 & $\begin{array}{l}\text { FAD-dependent } \\
\text { oxidoreductase, NADP } \\
\text { binding proteins }\end{array}$ \\
\hline 7 & $\begin{array}{l}\text { squalene } \\
\text { epoxidase } \\
\text { [Trichophyton } \\
\text { mentagrophytes] }\end{array}$ & QIJ32791.1 & $99 \%$ & $6 e-135$ & 44.69 & 489 & $\begin{array}{l}\text { FAD-dependent } \\
\text { oxidoreductase, NADP } \\
\text { binding proteins }\end{array}$ \\
\hline 8 & $\begin{array}{l}\text { squalene } \\
\text { epoxidase } \\
\text { [Trichophyton } \\
\text { mentagrophytes] }\end{array}$ & QHO61327.1 & $98 \%$ & $1 e-134$ & 44.66 & 487 & $\begin{array}{l}\text { FAD-dependent } \\
\text { oxidoreductase, NADP } \\
\text { binding proteins }\end{array}$ \\
\hline 9 & $\begin{array}{l}\text { squalene } \\
\text { epoxidase } \\
\text { [Trichophyton } \\
\text { mentagrophytes] }\end{array}$ & QGA31127.1 & $95 \%$ & $1 e-129$ & 44.82 & 453 & $\begin{array}{l}\text { FAD-dependent } \\
\text { oxidoreductase, NADP } \\
\text { binding proteins }\end{array}$ \\
\hline 10 & $\begin{array}{l}\text { squalene } \\
\text { epoxidase } \\
\text { [Trichophyton } \\
\text { mentagrophytes] }\end{array}$ & QIB02548.1 & $95 \%$ & $2 e-129$ & 44.82 & 453 & $\begin{array}{l}\text { FAD-dependent } \\
\text { oxidoreductase, NADP } \\
\text { binding proteins }\end{array}$ \\
\hline 11 & $\begin{array}{l}\text { squalene } \\
\text { epoxidase } \\
\text { [Trichophyton }\end{array}$ & $\begin{array}{c}\text { XP_00323384 } \\
5.1\end{array}$ & $99 \%$ & $5 e-136$ & 45.34 & 489 & $\begin{array}{l}\text { FAD-dependent } \\
\text { oxidoreductase, NADP } \\
\text { binding proteins }\end{array}$ \\
\hline
\end{tabular}




\begin{tabular}{|c|c|c|c|c|c|c|c|}
\hline & $\begin{array}{l}\text { rubrum CBS } \\
\text { 118892] }\end{array}$ & & & & & & \\
\hline 12 & $\begin{array}{l}\text { squalene } \\
\text { epoxidase } \\
\text { [Trichophyton } \\
\text { rubrum] }\end{array}$ & AAQ18216.1 & $99 \%$ & $1 e-135$ & 45.12 & 489 & $\begin{array}{l}\text { FAD-dependent } \\
\text { oxidoreductase, NADP } \\
\text { binding proteins }\end{array}$ \\
\hline 13 & $\begin{array}{l}\text { squalene } \\
\text { epoxidase } \\
\text { [Trichophyton } \\
\text { rubrum] }\end{array}$ & AAZ08563.1 & $99 \%$ & $4 e-135$ & 45.12 & 489 & $\begin{array}{l}\text { FAD-dependent } \\
\text { oxidoreductase, NADP } \\
\text { binding proteins }\end{array}$ \\
\hline 14 & $\begin{array}{l}\text { squalene } \\
\text { epoxidase } \\
\text { [Trichophyton } \\
\text { rubrum] }\end{array}$ & ATA67047.1 & $99 \%$ & $1 e-135$ & 44.90 & 489 & $\begin{array}{l}\text { FAD-dependent } \\
\text { oxidoreductase, NADP } \\
\text { binding proteins }\end{array}$ \\
\hline 15 & $\begin{array}{l}\text { squalene } \\
\text { epoxidase } \\
\text { [Trichophyton } \\
\text { rubrum] }\end{array}$ & ATA67045.1 & $99 \%$ & $2 e-135$ & 44.90 & 489 & $\begin{array}{l}\text { FAD-dependent } \\
\text { oxidoreductase, NADP } \\
\text { binding proteins }\end{array}$ \\
\hline 16 & $\begin{array}{l}\text { squalene } \\
\text { epoxidase } \\
\text { [Trichophyton } \\
\text { rubrum] }\end{array}$ & ATA67048.1 & $99 \%$ & $2 e-134$ & 44.69 & 489 & $\begin{array}{l}\text { FAD-dependent } \\
\text { oxidoreductase, NADP } \\
\text { binding proteins }\end{array}$ \\
\hline 17 & $\begin{array}{l}\text { squalene } \\
\text { epoxidase } \\
\text { [Trichophyton } \\
\text { interdigitale] }\end{array}$ & AVR57463.2 & $99 \%$ & $1 e-135$ & 44.90 & 489 & $\begin{array}{l}\text { FAD-dependent } \\
\text { oxidoreductase, NADP } \\
\text { binding proteins }\end{array}$ \\
\hline 18 & $\begin{array}{l}\text { squalene } \\
\text { epoxidase } \\
\text { [Trichophyton } \\
\text { interdigitale] }\end{array}$ & AVU05317.1 & $99 \%$ & $3 e-135$ & 44.69 & 489 & $\begin{array}{l}\text { FAD-dependent } \\
\text { oxidoreductase, NADP } \\
\text { binding proteins }\end{array}$ \\
\hline 19 & $\begin{array}{l}\text { squalene } \\
\text { epoxidase } \\
\text { [Trichophyton } \\
\text { interdigitale] }\end{array}$ & AVU05318.1 & $99 \%$ & $5 e-135$ & 44.69 & 489 & $\begin{array}{l}\text { FAD-dependent } \\
\text { oxidoreductase, NADP } \\
\text { binding proteins }\end{array}$ \\
\hline 20 & $\begin{array}{l}\text { squalene } \\
\text { epoxidase } \\
\text { [Trichophyton } \\
\text { interdigitale] }\end{array}$ & QBL54211.1 & $98 \%$ & $2 e-134$ & 44.66 & 487 & $\begin{array}{l}\text { FAD-dependent } \\
\text { oxidoreductase, } \\
\text { NADP binding } \\
\text { proteins }\end{array}$ \\
\hline 21 & $\begin{array}{l}\text { squalene } \\
\text { epoxidase } \\
\text { [Trichophyton } \\
\text { interdigitale] }\end{array}$ & QBL54209.1 & $98 \%$ & $5 e-134$ & 44.66 & 487 & $\begin{array}{l}\text { FAD-dependent } \\
\text { oxidoreductase, NADP } \\
\text { binding proteins }\end{array}$ \\
\hline 22 & $\begin{array}{l}\text { squalene } \\
\text { epoxidase } \\
\text { [Trichophyton } \\
\text { tonsurans CBS } \\
\text { 112818] }\end{array}$ & EGD99021.1 & $99 \%$ & $7 e-136$ & 44.90 & 489 & $\begin{array}{l}\text { FAD-dependent } \\
\text { oxidoreductase, NADP } \\
\text { binding proteins }\end{array}$ \\
\hline
\end{tabular}




\section{Prediction of exon count and number of transcripts}

An exon is a coding region of a gene that contains the information necessary to encode a protein. Numbers of exons of different species of dermatophytes were obtained from NCBI. A single gene can produce multiple different RNAs (transcripts). All squalene epoxidase genes generally contain 2 to 3 transcripts. Similarly, the numbers of transcripts were also obtained from NCBI's genomic regions, as shown in table 2 .

Table 2: Exon count and number of transcripts of paralogs of Squalene epoxidase gene.

\begin{tabular}{|c|c|c|c|c|}
\hline S. no & Species & Accession no & No of exons & Transcript no \\
\hline 1 & $\begin{array}{l}\text { squalene epoxidase } \\
\text { [Trichophyton mentagrophytes] }\end{array}$ & APQ47162.1 & 2 & 3 \\
\hline 2 & $\begin{array}{l}\text { squalene epoxidase } \\
\text { [Trichophyton mentagrophytes] }\end{array}$ & ATA67033.2 & 1 & 2 \\
\hline 3 & $\begin{array}{l}\text { squalene epoxidase } \\
\text { [Trichophyton mentagrophytes] }\end{array}$ & QIJ32792.1 & 2 & 3 \\
\hline 4 & $\begin{array}{l}\text { squalene epoxidase } \\
\text { [Trichophyton mentagrophytes] }\end{array}$ & QIJ32790.1 & 2 & 3 \\
\hline 5 & $\begin{array}{l}\text { squalene epoxidase } \\
\text { [Trichophyton mentagrophytes] }\end{array}$ & QIJ32789.1 & 2 & 3 \\
\hline 6 & $\begin{array}{l}\text { squalene epoxidase } \\
\text { [Trichophyton mentagrophytes] }\end{array}$ & ATA67030.2 & 1 & 2 \\
\hline 7 & $\begin{array}{l}\text { squalene epoxidase } \\
\text { [Trichophyton mentagrophytes] }\end{array}$ & QIJ32791.1 & 2 & 3 \\
\hline 8 & $\begin{array}{l}\text { squalene epoxidase } \\
\text { [Trichophyton mentagrophytes] }\end{array}$ & QHO61327.1 & 2 & 2 \\
\hline 9 & $\begin{array}{l}\text { squalene epoxidase } \\
\text { [Trichophyton mentagrophytes] }\end{array}$ & QGA31127.1 & 2 & 3 \\
\hline 10 & $\begin{array}{l}\text { squalene epoxidase } \\
\text { [Trichophyton mentagrophytes] }\end{array}$ & QIB02548.1 & 2 & 3 \\
\hline 11 & $\begin{array}{l}\text { squalene epoxidase } \\
\text { [Trichophyton rubrum CBS } \\
\text { 118892] }\end{array}$ & XP_003233845.1 & 2 & 3 \\
\hline 12 & $\begin{array}{l}\text { squalene epoxidase } \\
\text { [Trichophyton rubrum] }\end{array}$ & AAQ18216.1 & 2 & 2 \\
\hline 13 & $\begin{array}{l}\text { squalene epoxidase } \\
\text { [Trichophyton rubrum] }\end{array}$ & AAZ08563.1 & 2 & 3 \\
\hline 14 & $\begin{array}{l}\text { squalene epoxidase } \\
\text { [Trichophyton rubrum] }\end{array}$ & ATA67047.1 & 2 & 3 \\
\hline 15 & $\begin{array}{l}\text { squalene epoxidase } \\
\text { [Trichophyton rubrum] }\end{array}$ & ATA67045.1 & 2 & 3 \\
\hline 16 & $\begin{array}{l}\text { squalene epoxidase } \\
\text { [Trichophyton rubrum] }\end{array}$ & ATA67048.1 & 2 & 3 \\
\hline 17 & $\begin{array}{l}\text { squalene epoxidase } \\
\text { [Trichophyton interdigitale] }\end{array}$ & AVR57463.2 & 1 & 2 \\
\hline 18 & $\begin{array}{l}\text { squalene epoxidase } \\
\text { [Trichophyton interdigitale] }\end{array}$ & AVU05317.1 & 2 & 3 \\
\hline 19 & $\begin{array}{l}\text { squalene epoxidase } \\
\text { [Trichophyton interdigitale] }\end{array}$ & AVU05318.1 & 2 & 3 \\
\hline
\end{tabular}




\begin{tabular}{lllll}
\hline 20 & $\begin{array}{l}\text { squalene epoxidase } \\
\text { [Trichophyton interdigitale] }\end{array}$ & QBL54211.1 & 2 & 2 \\
\hline 21 & $\begin{array}{l}\text { squalene epoxidase } \\
{[\text { Trichophyton interdigitale] }}\end{array}$ & QBL54209.1 & 2 & 2 \\
\hline 22 & $\begin{array}{l}\text { squalene epoxidase } \\
\text { [Trichophyton tonsurans CBS } \\
\text { 112818] }\end{array}$ & EGD99021.1 & 2 & 3 \\
\hline
\end{tabular}

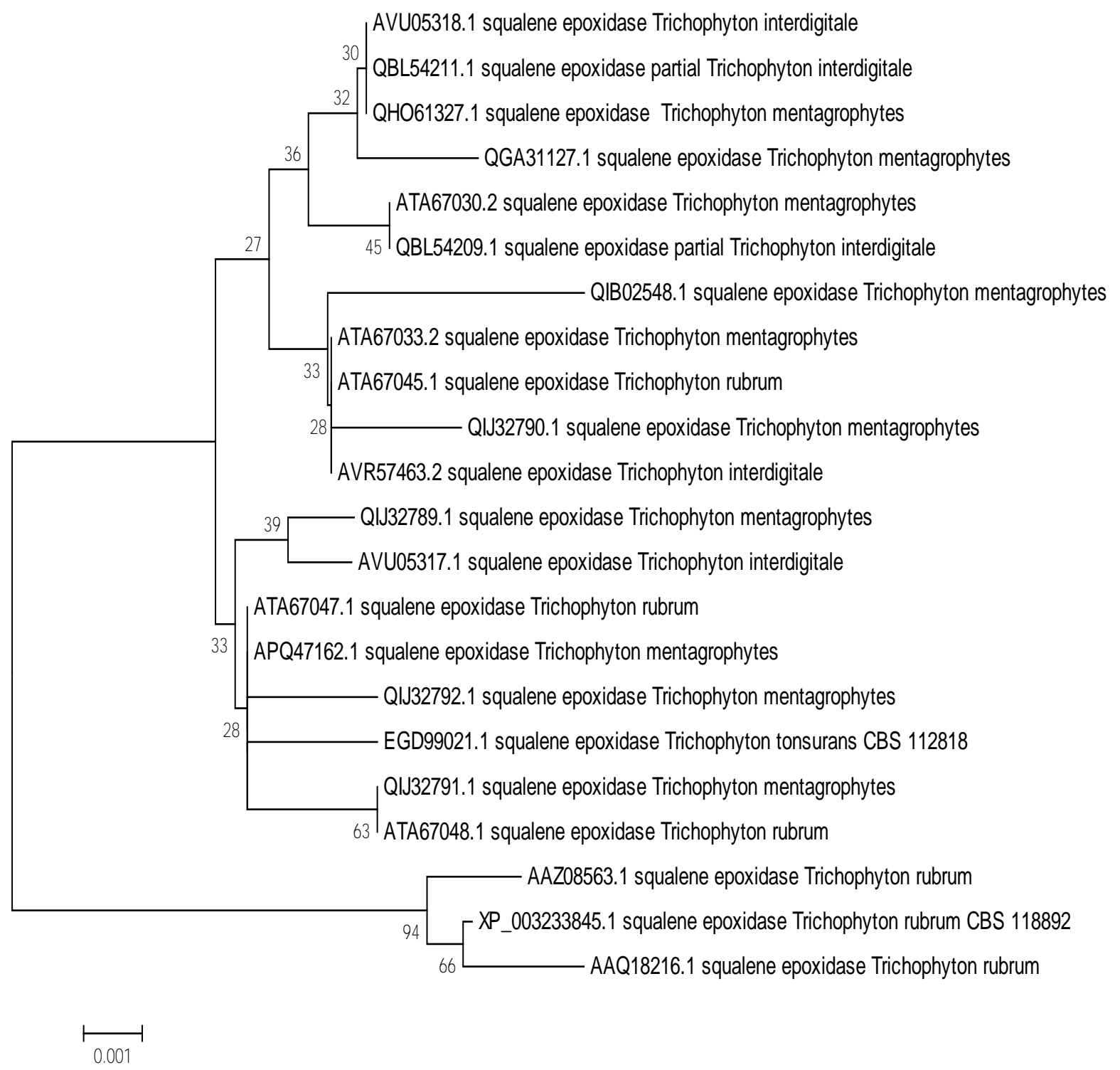

Figure 1: Multiple sequence alignment of amino acid sequences of 22 species were performed using clustalx. Phylogenetic tree of paralogs of squalene epoxidase protein sequences of various dermatophytes. The construction of the tree was performed with Mega 6. Sequences are annotated with their GenBank accession numbers. 


\section{Orthologs of Squalene epoxidase}

Similarly for orthologs, Squalene epoxidase gene was compared with Microsporum canis, Aspergillus versicolor, Aspergillus flavus, Aspergillus fumigatus, Aspergillus niger, Candida albicans and Candida glabrata. Conserved domain database search demonstrated the existence of conserved domain of squalene epoxidase gene. Another functional domain found was NADP binding domain, also recognized as Rossmann fold. Amino acid sequence length varies among different species from $405 \mathrm{bp}$ to $512 \mathrm{bp}$. For orthologs, Aspergillus fumigatus has highest similarity for ERG1. Percentage similarity was low compared to paralogs of ERG1, and query cover was $98 \%$, as shown in table 3 .

Table 3: Orthologs of Squalene epoxidase gene.

\begin{tabular}{|c|c|c|c|c|c|c|c|}
\hline $\begin{array}{l}\text { S. } \\
\text { no }\end{array}$ & Species name & Accession no. & $\begin{array}{l}\text { Query } \\
\text { cover }\end{array}$ & $\begin{array}{c}\text { E- } \\
\text { value }\end{array}$ & $\begin{array}{l}\text { Percentage } \\
\text { similarity }\end{array}$ & $\begin{array}{l}\text { Amino } \\
\text { acid } \\
\text { sequence } \\
\text { length }\end{array}$ & $\begin{array}{l}\text { Conserved } \\
\text { domains }\end{array}$ \\
\hline 1 & $\begin{array}{l}\text { squalene epoxidase } \\
\text { [Microsporum canis } \\
\text { CBS 113480] }\end{array}$ & $\begin{array}{c}\mathrm{XP} 002849431 . \\
1\end{array}$ & $84 \%$ & $1 e-106$ & 42.86 & 425 & $\begin{array}{l}\text { FAD-dependent } \\
\text { oxidoreductases, } \\
\text { NADP binding } \\
\text { proteins }\end{array}$ \\
\hline 2 & $\begin{array}{l}\text { squalene epoxidase } \\
\text { [Microsporum canis] }\end{array}$ & BBC18146.1 & $84 \%$ & $4 e-105$ & 42.35 & 425 & $\begin{array}{l}\text { FAD-dependent } \\
\text { oxidoreductases, } \\
\text { NADP binding } \\
\text { proteins }\end{array}$ \\
\hline 3 & $\begin{array}{l}\text { squalene } \\
\text { monooxygenase } \\
\text { [Nannizzia gypsea } \\
\text { CBS 118893] }\end{array}$ & XP_003170113.1 & $99 \%$ & $2 e-134$ & 44.57 & 489 & $\begin{array}{l}\text { FAD-dependent } \\
\text { oxidoreductases, } \\
\text { NADP binding } \\
\text { proteins }\end{array}$ \\
\hline 4 & $\begin{array}{l}\text { hypothetical protein } \\
\text { ASPVEDRAFT_52898 } \\
\text { [Aspergillus versicolor } \\
\text { CBS 583.65] }\end{array}$ & OJJ02152.1 & $98 \%$ & $1 e-114$ & 43.11 & 1427 & $\begin{array}{l}\text { FAD-dependent } \\
\text { oxidoreductases, } \\
\text { NADP binding } \\
\text { proteins }\end{array}$ \\
\hline 5 & $\begin{array}{l}\text { squalene } \\
\text { monooxygenase Erg1 } \\
\text { [Aspergillus flavus } \\
\text { NRRL3357] }\end{array}$ & $\begin{array}{c}\text { XP_002378894. } \\
1\end{array}$ & $98 \%$ & $1 e-128$ & 42.98 & 480 & $\begin{array}{l}\text { FAD-dependent } \\
\text { oxidoreductases, } \\
\text { NADP binding } \\
\text { proteins }\end{array}$ \\
\hline 6 & $\begin{array}{l}\text { squalene epoxidase } \\
\text { [Aspergillus flavus] }\end{array}$ & RAQ67502.1 & $98 \%$ & $2 e-128$ & 42.98 & 480 & $\begin{array}{l}\text { FAD-dependent } \\
\text { oxidoreductases, } \\
\text { NADP binding } \\
\text { proteins }\end{array}$ \\
\hline 7 & $\begin{array}{l}\text { squalene } \\
\text { monooxygenase Erg1 } \\
\text { [Aspergillus flavus } \\
\text { AF70] }\end{array}$ & KOC12150.1 & $98 \%$ & $4 e-128$ & 42.76 & 480 & $\begin{array}{l}\text { FAD-dependent } \\
\text { oxidoreductases, } \\
\text { NADP binding } \\
\text { proteins }\end{array}$ \\
\hline 8 & $\begin{array}{l}\text { squalene epoxidase } \\
\text { [Aspergillus flavus] }\end{array}$ & RAQ47162.1 & $98 \%$ & $8 e-128$ & 42.76 & 480 & $\begin{array}{l}\text { FAD-dependent } \\
\text { oxidoreductases, } \\
\text { NADP binding } \\
\text { proteins }\end{array}$ \\
\hline 9 & $\begin{array}{l}\text { squalene } \\
\text { monooxygenase Erg1 }\end{array}$ & XP_753858.1 & $98 \%$ & $2 e-130$ & 44.93 & 481 & $\begin{array}{l}\text { FAD-dependent } \\
\text { oxidoreductases, }\end{array}$ \\
\hline
\end{tabular}




\begin{tabular}{|c|c|c|c|c|c|c|c|}
\hline & $\begin{array}{l}\text { [Aspergillus } \\
\text { fumigatus Af293] }\end{array}$ & & & & & & $\begin{array}{l}\text { NADP binding } \\
\text { proteins }\end{array}$ \\
\hline 10 & $\begin{array}{l}\text { squalene } \\
\text { monooxygenase Erg1 } \\
\text { [Aspergillus } \\
\text { fumigatus Z5] }\end{array}$ & KMK59426.1 & $98 \%$ & $4 e-130$ & 44.71 & 481 & $\begin{array}{l}\text { FAD-dependent } \\
\text { oxidoreductases, } \\
\text { NADP binding } \\
\text { proteins }\end{array}$ \\
\hline 11 & $\begin{array}{l}\text { hypothetical protein } \\
\text { CNMCM8812_000993 } \\
\text { [Aspergillus } \\
\text { fumigatus] }\end{array}$ & KAF4275551.1 & $98 \%$ & $5 e-128$ & 44.93 & 481 & $\begin{array}{l}\text { FAD-dependent } \\
\text { oxidoreductases, } \\
\text { NADP binding } \\
\text { proteins }\end{array}$ \\
\hline 12 & $\begin{array}{l}\text { squalene epoxidase } 1 \\
\text { [Aspergillus } \\
\text { fumigatus] }\end{array}$ & AAS60234.1 & $79 \%$ & $5 e-106$ & 45.53 & 405 & $\begin{array}{l}\text { FAD-dependent } \\
\text { oxidoreductases, } \\
\text { NADP binding } \\
\text { proteins }\end{array}$ \\
\hline 13 & $\begin{array}{l}\text { squalene epoxidase } \\
\text { [Aspergillus niger } \\
\text { CBS 513.88] }\end{array}$ & $\begin{array}{c}\text { XP_001390258. } \\
1\end{array}$ & $98 \%$ & $2 e-130$ & 45.41 & 512 & $\begin{array}{l}\text { FAD-dependent } \\
\text { oxidoreductases, } \\
\text { NADP binding } \\
\text { proteins }\end{array}$ \\
\hline 14 & $\begin{array}{l}\text { SE-domain- } \\
\text { containing protein } \\
\text { [Aspergillus niger } \\
\text { CBS 101883] }\end{array}$ & $\begin{array}{c}\text { XP_025456429. } \\
1\end{array}$ & $98 \%$ & $8 e-131$ & 45.41 & 478 & $\begin{array}{l}\text { FAD-dependent } \\
\text { oxidoreductases, } \\
\text { NADP binding } \\
\text { proteins }\end{array}$ \\
\hline 15 & $\begin{array}{l}\text { squalene } \\
\text { monooxygenase Erg1 } \\
\text { [Aspergillus fischeri } \\
\text { NRRL 181] }\end{array}$ & $\begin{array}{c}\text { XP_001259905. } \\
1\end{array}$ & $98 \%$ & $1 e-126$ & 44.93 & 481 & $\begin{array}{l}\text { FAD-dependent } \\
\text { oxidoreductases, } \\
\text { NADP binding } \\
\text { proteins }\end{array}$ \\
\hline 16 & $\begin{array}{l}\text { squalene } \\
\text { monooxygenase } \\
\text { [Candida albicans } \\
\text { P94015] }\end{array}$ & KGQ90519.1 & $99 \%$ & $3 e-115$ & 38.71 & 496 & $\begin{array}{l}\text { FAD-dependent } \\
\text { oxidoreductases, } \\
\text { NADP binding } \\
\text { proteins }\end{array}$ \\
\hline 17 & $\begin{array}{l}\text { squalene } \\
\text { monooxygenase } \\
\text { [Candida albicans } \\
\text { SC5314] }\end{array}$ & XP_711894.1 & $99 \%$ & $3 e-115$ & 38.71 & 496 & $\begin{array}{l}\text { FAD-dependent } \\
\text { oxidoreductases, } \\
\text { NADP binding } \\
\text { proteins }\end{array}$ \\
\hline 18 & $\begin{array}{l}\text { squalene } \\
\text { monooxygenase } \\
\text { [Candida albicans } \\
\text { Ca529L] }\end{array}$ & RLP62132.1 & $99 \%$ & $3 e-115$ & 38.71 & 496 & $\begin{array}{l}\text { FAD-dependent } \\
\text { oxidoreductases, } \\
\text { NADP binding } \\
\text { proteins }\end{array}$ \\
\hline 19 & $\begin{array}{l}\text { uncharacterized } \\
\text { protein } \\
\text { CAGL0D05940g } \\
\text { [[Candida] glabrata] }\end{array}$ & XP_445667.1 & $98 \%$ & $1 e-106$ & 37.34 & 489 & $\begin{array}{l}\text { FAD-dependent } \\
\text { oxidoreductases, } \\
\text { NADP binding } \\
\text { proteins }\end{array}$ \\
\hline 20 & $\begin{array}{l}\text { squalene } \\
\text { monooxygenase } \\
\text { [Candida tropicalis } \\
\text { MYA-3404] }\end{array}$ & XP_002551185.1 & $98 \%$ & $9 e-115$ & 38.98 & 497 & $\begin{array}{l}\text { FAD-dependent } \\
\text { oxidoreductases, } \\
\text { NADP binding } \\
\text { proteins }\end{array}$ \\
\hline
\end{tabular}




\section{Predicting exon count, a number of transcripts and chromosome location.}

Numbers of exons were obtained from NCBI from a genomic context. All aspergillus species have only one gene transcript. Numbers of transcripts were also obtained from genomic regions from NCBI. The chromosome of almost all species waswas unknown except Aspergillus fumigatus, located on chromosome no 5 and Candida albicans are located on chromosome no 1 as shown in table 4.

Table 4: Exon count, number of transcripts and chromosomal location of orthologs of Squalene epoxidase gene.

\begin{tabular}{|c|c|c|c|c|c|}
\hline $\begin{array}{l}\text { S. } \\
\text { no }\end{array}$ & Species & Accession no & $\begin{array}{l}\text { No of } \\
\text { exons }\end{array}$ & $\begin{array}{c}\text { No of } \\
\text { transcripts }\end{array}$ & Chromosome no \\
\hline 1 & $\begin{array}{l}\text { squalene epoxidase } \\
\text { [Microsporum canis CBS 113480] }\end{array}$ & XP_002849431.1 & 3 & 2 & Unknown \\
\hline 2 & $\begin{array}{l}\text { squalene epoxidase } \\
\text { [Microsporum canis] }\end{array}$ & BBC18146.1 & 3 & 2 & Unknown \\
\hline 3 & $\begin{array}{l}\text { squalene monooxygenase } \\
\text { [Nannizzia gypsea CBS 118893] }\end{array}$ & XP_003170113.1 & 2 & 2 & Unknown \\
\hline 4 & $\begin{array}{l}\text { hypothetical protein } \\
\text { ASPVEDRAFT_52898 } \\
\text { [Aspergillus Versicolor CBS } \\
583.65 \text { ] }\end{array}$ & OJJ02152.1 & 3 & 1 & Unknown \\
\hline 5 & $\begin{array}{l}\text { squalene monooxygenase Erg1 } \\
\text { [Aspergillusflavus NRRL3357] }\end{array}$ & XP_002378894.1 & 2 & 1 & Unknown \\
\hline 6 & $\begin{array}{l}\text { squalene epoxidase [Aspergillus } \\
\text { flavus] }\end{array}$ & RAQ67502.1 & 2 & 1 & Unknown \\
\hline 7 & $\begin{array}{l}\text { squalene monooxygenase Erg1 } \\
\text { [Aspergillus flavus AF70] }\end{array}$ & KOC12150.1 & 2 & 1 & Unknown \\
\hline 8 & $\begin{array}{l}\text { squaleneepoxidase [Aspergillus } \\
\text { flavus] }\end{array}$ & RAQ47162.1 & 2 & 1 & Unknown \\
\hline 9 & $\begin{array}{l}\text { squalene monooxygenase Erg1 } \\
\text { [Aspergillus fumigatus Af293] }\end{array}$ & XP_753858.1 & 2 & 1 & Chromosome 5 \\
\hline 10 & $\begin{array}{l}\text { squalene monooxygenase Erg1 } \\
\text { [Aspergillus fumigatus Z5] }\end{array}$ & KMK59426.1 & 2 & 1 & Chromosome 5 \\
\hline 11 & $\begin{array}{l}\text { hypothetical protein } \\
\text { CNMCM8812_000993 } \\
\text { [Aspergillus fumigatus] }\end{array}$ & KAF4275551.1 & 2 & 1 & Unknown \\
\hline 12 & $\begin{array}{l}\text { squalene epoxidase } 1 \\
\text { [Aspergillus fumigatus] }\end{array}$ & AAS60234.1 & 2 & 1 & Unknown \\
\hline 13 & $\begin{array}{l}\text { squalene epoxidase [Aspergillus } \\
\text { niger CBS 513.88] }\end{array}$ & XP_001390258.1 & 2 & 1 & Unknown \\
\hline 14 & $\begin{array}{l}\text { SE-domain-containing protein } \\
\text { [Aspergillus niger CBS 101883] }\end{array}$ & XP_025456429.1 & 2 & 1 & Unknown \\
\hline 15 & $\begin{array}{l}\text { squalene monooxygenase Erg1 } \\
\text { [Aspergillus fischeri NRRL 181] }\end{array}$ & XP_001259905.1 & 2 & 1 & Unknown \\
\hline 16 & $\begin{array}{l}\text { squalene monooxygenase } \\
\text { [Candida albicans P94015] }\end{array}$ & KGQ90519.1 & 1 & 1 & Chromosome 1 \\
\hline
\end{tabular}




\begin{tabular}{llcccc}
\hline 17 & $\begin{array}{l}\text { squalene monooxygenase } \\
\text { [Candida albicans SC5314] }\end{array}$ & XP_711894.1 & 1 & 1 & Chromosome 1 \\
\hline 18 & $\begin{array}{l}\text { squalene monooxygenase } \\
\text { [Candida albicans Ca529L }\end{array}$ & RLP62132.1 & 1 & 1 & Chromosome 1 \\
\hline 19 & $\begin{array}{l}\text { uncharacterized protein } \\
\text { CAGLOD05940g [[Candida] } \\
\text { glabrata] }\end{array}$ & XP_445667.1 & 1 & 1 & Chromosome D \\
\hline 20 & $\begin{array}{l}\text { squalene monooxygenase } \\
\text { [Candida tropicalis MYA-3404] }\end{array}$ & XP_002551185.1 & 1 & 1 & Unknown \\
\hline
\end{tabular}

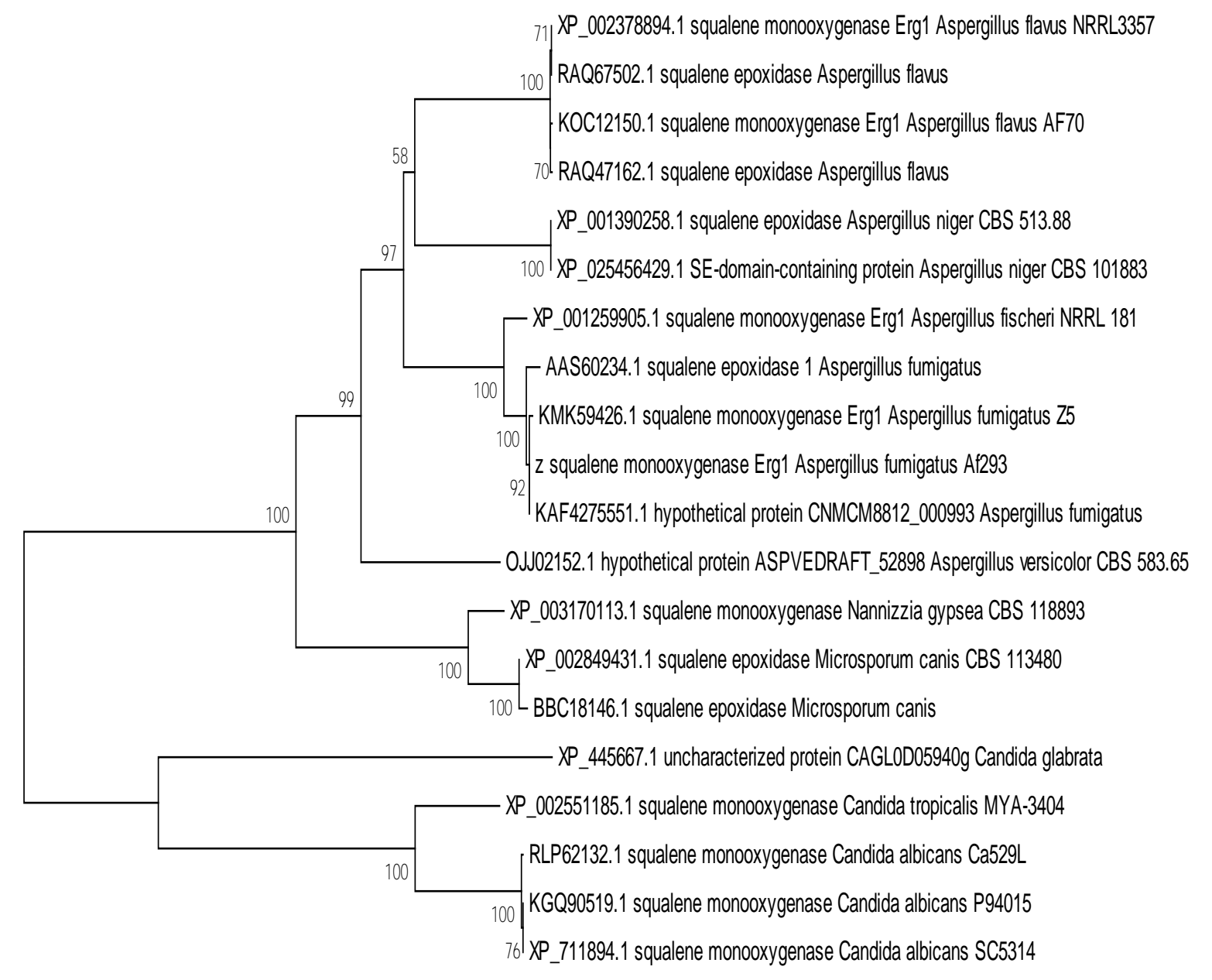

0.1

Figure 2: Phylogenetic tree of orthologs of squalene epoxidase amino acid sequences. The tree was constructed using the neighbour-joining method in MEGA6 software. Protein sequences were obtained from the Gene bank. 


\section{Single nucleotide polymorphisms (SNPs) in Squalene epoxidase gene}

Single nucleotide polymorphisms (SNPs) have been identified in the squalene epoxidase gene of the Trichophyton group. Resistance in the gene encoding squalene epoxidase is attributable to point mutations. The mutation was observed in Trichophyton interdigital and Trichophyton rubrum isolates. Trichophyton interdigitale nucleotide substitution in the Squalene epoxidase gene was CTC, which was substituted into TTC at 1184. Similarly, another substitution was found at position 1185, leading to the replacement of TTC by CTC. For Trichophyton rubrum, a substitution occurred at position 1380, leading to the replacement of CAT by TAT. Another substitution was at position 1175, leading to the replacement of TTC by TTA in the squalene epoxidase gene, as shown in table 5.

Table 5. SNPs in Squalene epoxidase gene of Trichophyton group.

\begin{tabular}{|c|c|c|c|c|}
\hline Species & Accession no & $\begin{array}{l}\text { Nucleotide } \\
\text { substitution in } \\
\text { SQLE gene }\end{array}$ & $\begin{array}{l}\text { SNPs in this } \\
\text { study }\end{array}$ & $\begin{array}{c}\text { Reported } \\
12 \text { (Burmester, } \\
\text { Hipler et al. 2020) }\end{array}$ \\
\hline $\begin{array}{l}\text { Squalene epoxidase } \\
\text { [Trichophyton } \\
\text { mentagrophytes] }\end{array}$ & МT371234.1 & $\mathrm{TTG} \rightarrow \mathrm{TTC}$ & 1184 & 1189 \\
\hline $\begin{array}{l}\text { Squalene epoxidase } \\
\text { [Trichophyton } \\
\text { mentagrophytes] }\end{array}$ & МT371233.1 & $\mathrm{TTG} \rightarrow \mathrm{TTC}$ & 1184 & 1189 \\
\hline $\begin{array}{l}\text { Squalene epoxidase } \\
\text { [Trichophyton } \\
\text { mentagrophytes ] }\end{array}$ & MT371235.1 & $\mathrm{CTC} \rightarrow \mathrm{TTC}$ & 1184 & 1189 \\
\hline $\begin{array}{l}\text { squalene epoxidase, partial } \\
\text { [Trichophyton interdigitale] }\end{array}$ & QBL54211.1 & $\mathrm{TTC} \rightarrow \mathrm{CTC}$ & 1185 & 1189 \\
\hline $\begin{array}{l}\text { squalene epoxidase, partial } \\
\text { [Trichophyton interdigitale] }\end{array}$ & QBL54209.1 & $\mathrm{TTC} \rightarrow \mathrm{CTC}$ & 1185 & 1189 \\
\hline $\begin{array}{l}\text { squalene epoxidase } \\
\text { [Trichophyton rubrum] }\end{array}$ & AAQ18216.1 & $\mathrm{CAT} \rightarrow \mathrm{TAT}$ & 1380 & 1380 \\
\hline $\begin{array}{l}\text { squalene epoxidase } \\
\text { [Trichophyton rubrum] }\end{array}$ & AAZ08563.1 & $\mathrm{TTT} \rightarrow \mathrm{TTA}$ & 1197 & 1177 \\
\hline $\begin{array}{l}\text { squalene epoxidase } \\
\text { [Trichophyton rubrum] }\end{array}$ & ATA67047.1 & $\mathrm{TTC} \rightarrow \mathrm{TTA}$ & 1175 & 1189 \\
\hline $\begin{array}{l}\text { squalene epoxidase } \\
\text { [Trichophyton rubrum] }\end{array}$ & ATA67045.1 & $\mathrm{TTC} \rightarrow \mathrm{TTA}$ & 1175 & 1189 \\
\hline $\begin{array}{l}\text { squalene epoxidase } \\
\text { [Trichophyton rubrum] }\end{array}$ & ATA67048.1 & $\mathrm{TAT} \rightarrow \mathrm{CAT}$ & 1368 & 1380 \\
\hline
\end{tabular}

\section{Discussion}

Squalene epoxidase is a monooxygenase that catalyzes the conversion of squalene to 2,3 oxidosqualene. Terbinafine inhibits squalene epoxidase, a key enzyme in the ergosterol biosynthesis pathway. This inhibition contributes to squalene aggregation, ergosterol depletion and the inhibition of growth of fungus ${ }^{13}$. This study was focused on the abundance of squalene epoxidase in dermatophytes. Gene sequence Percentage similarity ranges were suggested from $44.66 \%$ 45.34\%. Query cover ranges from 95\% - 99\%. The percentage similarity of Trichophyton rubrum was higher than other species of dermatophytes such as Trichophyton. Trichophyton mentagrophytes have one exon, while all other species have two exons. The number of transcripts was 2-3 for all species. Chromosomal locations of all species were 
unknown except Aspergillus fumigatus was located on chromosome no $5^{14}$.

The number of detected genes contained FAD and NABD binding domain, which was similar in all genomes. These domains have an alpha-betaalpha configuration. NAD binding involves numerous hydrogen, and van der Waals contacts ${ }^{15}$. Trichophyton rubrum has a percentage similarity of 45.12 with the squalene epoxidase gene. For orthologs, Aspergillus fumigatus has the highest percentage similarity ${ }^{16}$. The bioinformatics survey of the squalene epoxidase gene revealed that dermatophytes comprise a closely-related and diverged lineage of the squalene epoxidase gene. A variety of divergence of squalene epoxidase gene was found in three fungal groups of dermatophytes, while squalene epoxidase homologs were not found in Epidermophyton ${ }^{17}$.

Single-nucleotide polymorphism (SNP) is a substitution of a single nucleotide at a specific genome position. Single nucleotide polymorphisms occur within coding sequences of genes, non-coding regions of genes, or in the intergenic regions between genes. SNPs in the coding regions are of two kinds synonymous and non-synonymous SNPs. Synonymous SNPs do not affect protein or DNA sequence, while nonsynonymous SNPs change the amino acid sequence of protein or DNA ${ }^{18}$. SNPs have been identified in squalene epoxidase homologs of Trichophyton group ${ }^{19}$. Nucleotide substitution within the squalene epoxidase gene was observed in Trichophyton interdigitale all isolates that substitutes TTC with CTC at 1185 position ${ }^{20}$. In Trichophyton rubrum, nucleotide substitution occurred at 1380, leading to the replacement of CAT with TAT $^{8}$. Another substitution occurred at 1175 position leading to the replacement of TTC by TTA that was near related to previous study ${ }^{21}$ in which the nucleotide and amino acid substitution in the squalene epoxidase gene varies between homologs of the Trichophyton group.

\section{Conclusion}

Squalene epoxidase is an antifungal targeted key enzyme in the ergosterol biosynthesis pathway of several fungal groups. This inhibition of the gene contributes to squalene aggregation, ergosterol depletion and the inhibition of fungal growth. Studies have demonstrated a variety of squalene epoxidase genes in a major group of dermatophytes. Squalene epoxidase has widely diverged in three fungal groups of dermatophytes Trichophyton, Epidermophyton and Microsporum. Mutation in the squalene epoxidase gene can mislead normal sterol formation in the fungal cell membrane that induces fungal resistance against several drugs, including azoles and polyenes. Squalene epoxidase gene carries 2 - 3 transcripts and $2-3$ number of exons in dermatophytes. Squalene epoxidase gene has FAD-dependent oxidoreductases and NADP binding conserved domains similar in three major fungal groups of dermatophytes. Squalene epoxidase gene was also studied for possible single nucleotide polymorphism (SNPs) in the Trichophyton group's homologs and found multiple SNP positions on 1184, 1185 1380, 1175 and 1368 nucleotide positions of TTC $\rightarrow$ CTC. This position was the cause of nonsynonymous mutation, which can change the sequence of amino acid into a protein structure that can change phenylalanine to leucine.

\section{Conflicts of Interest}

None.

\section{Acknowledgement}

The authors would like to acknowledge Dr. Sadaf Ambreen for the technical support.

\section{Funding}

None.

\section{References}

1. Teklebirhan G, Bitew A. Prevalence of Dermatophytic Infection and the Spectrum of Dermatophytes in Patients Attending a Tertiary Hospital in Addis Ababa, Ethiopia. Int J Microbiol. 2015;653419(10):13.

2. White TC, Oliver BG, Gräser Y, Henn MR. Generating and testing molecular hypotheses in the dermatophytes. Eukaryot Cell. 2008;7(8):1238-1245. 
3. Motavaze K, Namvar Z, Emami M, Noorbakhsh F, Rezaie S. Molecular characterization of a Squalene epoxidase gene in dermatophyte pathogen Trichophyton tonsurans. Iran Biomed J. 2008;12(1):55-58.

4. Saunte DM, Hare RK, Jørgensen KM, Jørgensen R, Deleuran M, Zachariae CO, Thomsen SF, BjørnskovHalkier L, Kofoed K, Arendrup MC. Emerging Terbinafine Resistance $<$ em $>$ Trichophyton $</$ em $>$ : Clinical Characteristics, Squalene Epoxidase Gene Mutations, and a Reliable EUCAST Method for Detection. Antimicrob Agents Chemother. 2019;63(10):e01126-19.

5. Osborne CS, Leitner I, Favre B, Ryder NS. Amino Acid Substitution in <em>Trichophyton rubrum</em > Squalene Epoxidase Associated with Resistance to Terbinafine. Antimicrob Agents Chemother. 2005;49(7):2840-2844.

6. Martinez-Rossi NM, Peres NT, Rossi A. Antifungal resistance mechanisms in dermatophytes. Mycopathologia. 2008;166(5-6):369-383.

7. Yamada T, Maeda M, Alshahni MM, Tanaka R, Yaguchi T, Bontems O, Salamin K, Fratti M, Monod M. Terbinafine Resistance of Trichophyton Clinical Isolates Caused by Specific Point Mutations in the Squalene Epoxidase Gene. Antimicrob Agents Chemother. 2017;61(7):00115-17.

8. Rudramurthy SM, Shankarnarayan SA, Dogra S, Shaw D, Mushtaq K, Paul RA, Narang T, Chakrabarti A. mutation in the Squalene Epoxidase Gene of Trichophyton interdigitale and Trichophyton rubrum Associated with Allylamine Resistance. Antimicrob Agents Chemother. 2018;62(5):0252217.

9. Garaiová M, Zambojová V, Simová Z, Griač P, Hapala I. Squalene epoxidase as a target for manipulation of squalene levels in the yeast Saccharomyces cerevisiae. FEMS Yeast Res. 2014;14(2):310-323.

10. Padyana AK, Gross $S$, Jin L, Cianchetta $G$, Narayanaswamy R, Wang F, Wang R, Fang C, Lv X, Biller SA, Dang L. Structure and inhibition mechanism of the catalytic domain of human squalene epoxidase. Nat Commun. 2019;10(1):01807928.

11. Ruckenstuhl C, Eidenberger A, Lang S, Turnowsky F. Single amino acid exchanges in FAD-binding domains of squalene epoxidase of Saccharomyces cerevisiae lead to either loss of functionality or terbinafine sensitivity. Biochem. Soc. Trans. 2005;33:1197-1201.

12. Yamada T, Maeda M, Alshahni MM, Tanaka R, Yaguchi T, Bontems O, Salamin K, Fratti M, Monod
M. Terbinafine Resistance of Trichophyton Clinical Isolates Caused by Specific Point Mutations in the Squalene Epoxidase Gene. Antimicrob Agents Chemother. 2017;61(7).

13. Nowosielski M, Hoffmann M, Wyrwicz LS, Stepniak P, Plewczynski DM, Lazniewski M, Ginalski K, Rychlewski L. Detailed Mechanism of Squalene Epoxidase Inhibition by Terbinafine. J. Chem. Inf. Model. 2011;51:455-462.

14. Singh A, Masih A, Khurana A, Singh PK, Gupta M, Hagen F, Meis JF, Chowdhary A. High terbinafine resistance in Trichophyton interdigitale isolates in Delhi, India harbouring mutations in the squalene epoxidase gene. Mycoses. 2018;61(7):477-484.

15. Unland K, Pütter KM, Vorwerk K, van Deenen $N$, Twyman RM, Prüfer D, Schulze Gronover C. Functional characterization of squalene synthase and squalene epoxidase in Taraxacum koksaghyz. Plant Dir. 2018;2(6):e00063.

16. Ding C, Zhao CL, Chen ZJ, Wei FG, Song DG, Miao $K R$. Bioinformatics analysis of the squalene synthase gene and the amino acid sequence in ginseng species. Int J Clin Exp Med. 2015;8(8):12818-12825.

17. Ruckenstuhl C, Lang S, Poschenel A, Eidenberger A, Baral PK, Kohút P, Hapala I, Gruber K, Turnowsky F. Characterization of Squalene Epoxidase of $<$ em $>$ Saccharomyces cerevisiae $</$ em $>$ by Applying Terbinafine-Sensitive Variants. Antimicrob Agents Chemother. 2007;51(1):275-284.

18. Su L, Liu G, Wang H, Tian Y, Zhou Z, Han L, Yan L. Research on single nucleotide polymorphisms interaction detection from network perspective. PLoS One. 2015; 10(3):e0119146

19. Pchelin IM, Azarov DV, Chilina GA, Dmitriev KA, Vasilyeva NV, Taraskina AE. Single-nucleotide polymorphism in a local population of Trichophyton rubrum. Med Mycol. 2018;56(1):125-128.

20. Shankarnarayan SA, Shaw D, Sharma A, Chakrabarti A, Dogra S, Kumaran MS, Kaur H, Ghosh A, Rudramurthy SM. Rapid detection of terbinafine resistance in Trichophyton species by Amplified refractory mutation system-polymerase chain reaction. Scientific Reports. 2020;10(1):1297.

21. Burmester $A$, Hipler UC, Uhrlaß $S$, Nenoff $P$, Singal A, Verma SB, Elsner $P$, Wiegand C. Indian Trichophyton mentagrophytes squalene epoxidase erg1 double mutants show high proportion of combined fluconazole and terbinafine resistance. Mycoses. 2020;63(11):1175-1180. 